\title{
A novel coumarin-based compound inhibits invasion and migration of murine osteosarcoma cells in vitro
}

\author{
Yuki Sugiyama1, Seikou Nakamura ${ }^{1}$, Hiroki Fukuda², Masato Yoshizawa ${ }^{1}$, Shiori Tamai ${ }^{1}$, \\ Yuki Toda ${ }^{1}$, Kazuyuki Takata', Eishi Ashihara ${ }^{1}$ \\ ${ }^{I}$ Department of Clinical and Translational Physiology, Kyoto Pharmaceutical University, Japan, ${ }^{2}$ Department of \\ Pharmacognosy, Kyoto Pharmaceutical University, Japan
}

[INTRODUCTION] There are many molecular targeted agents for cancer developed so far. However we have few effective agents against metastasis. Without metastases, the five-year overall survivals and event-free survivals become approximately $70 \%$ and $60 \%$, respectively, in the patients with osteosarcoma. Whereas the five-year overall survivals and event-free survivals with metastases decreases to about $30 \%$ and $15 \%$, respectively. Therefore, it is important to develop new drugs that inhibit metastasis of cancer. We investigated the inhibitory effects of coumarin-based compounds against migration and invasion of murine LM-8 osteosarcoma cells in vitro.

[METHODS] We conducted a screen of coumarin-based compounds for inhibitors against invasion and migration of LM -8 cells using a transwell chamber system. Cell cytotoxicity was measured using a WST-8 assay. Alternations of protein expression after compound treatment were assessed by Western blotting analysis and immunofluorescent analysis. Quantitative RT-PCR analysis was conducted to investigate alternations of mRNA transcripts.

[RESULTS and DISCUSSION] Through a screen of coumarin-based compounds, we found that daphnetin inhibited the migration and invasion of LM-8 cells in a dose-dependent manner. Immunofluorescence analysis revealed that daphnetin decreased the number of intracellular stress fibers and filopodia. Moreover, daphnetin treatment dramatically decreased the expression levels of small GTP-binding proteins, RhoA and Cdc42. We performed a structure-activity relationship analysis and we developed a novel compound KPU-Shoyaku-192 (compound \#192). As with daphnetin, KPU-Shoyaku -192 inhibited invasion of LM-8 cells in a dose dependent manner without reduce growth rate and actin stress fibers and filopodia were diminished, and small GTP-binding protein levels were decreased in a dose-dependent manner by the treatment of compound \#192. Interestingly, compound \#192 did not alter the mRNA transcript levels of small GTPbinding proteins.

[CONCLUSION] A novel coumarin-based compound \#192 inhibits invasion and migration of LM-8 cells. Further investigations are carried out.

[REFERENCE] (1) Fukuda H, et al. Biochem Biophys Res Commun, 471, 63-67, 2016. 